\title{
Anaesthesiology in the undergraduate medical curriculum
}

\section{W Shane Journeay \\ Class of 2011, Faculty of Medicine, Dalhousie University}

A naesthesiology is a specialty that many medical students do not discover until late in their undergraduate training. For some it is a positive discovery in their clinical training leading to a rapid change in career direction. With a plethora of subspecialties for undergraduate medical students to explore in a short time, there is a paucity of exposure to anaesthesiology in the modern medical curriculum. Without early clinical or theoretical education of the role of the anaesthesiologist it is no wonder many medical students are left with an uninformed opinion of the specialty. Commonly a medical student's perspective of anaesthesiology is realized indirectly through the eyes of a surgical rotation.

At the core of an anaesthesiologist's broad skill set is the sound application of physiology and pharmacology. These two disciplines are intertwined and while they form the basis of effective anaesthesia and critical care medicine, education in these areas can be beneficial to a wide range of other specialities including surgery, emergency medicine and internal medicine. Indeed, there are basic concepts and skills in anaesthesiology in which every physician should possess, such as airway management. ${ }^{1}$ Anaesthesiology also presents some exciting new research opportunities in the area of patient safety, pain management and drug delivery using cutting edge technology. Perioperative care is also an area ideally suited for anaesthesiologists to lead in educating medical trainees. It is for these reasons that anaesthesiologists could play a more prominent role in modern medical curriculum. The following discussion will outline three key areas in which anaesthesiologists can contribute to undergraduate medical education.

\section{Anaesthesiologists as Leaders in Problem-Based Learning}

As many modern medical schools use problem-oriented teaching, the anaesthesiologist is ideally suited to link basic science curriculum to clinical scenarios. They would be particularly well equipped to provide expertise and input in the teaching of pharmacology and physiology. Indeed anaesthesiologists have been conducting problem-based learning sessions for students and residents for many years in the daily setting of the operating room or through various forms of rounds. ${ }^{2}$ Moreover they can enrich the curriculum given that they play a central role in a number of hospital settings including the OR, PACU, ICU, obstetrics, pain management, and pre-admission. A recent study demonstrated that of the 1089 faculty anaesthesiologists in Canada, only 53 are involved in problem-based learning tutorials, while an even lesser proportion (ten) are involved in writing of problem-based learning cases. ${ }^{3}$

The role of pharmacology application should be highlighted given the scarcity of direct clinical pharmacology training at medical schools in Canada. In fact only four medical schools offer clinical pharmacology in undergraduate curriculum. ${ }^{4}$ Additionally, four schools in Canada do not provide mandatory clinical rotations in anaesthesiology. In light of some of the more challenging subject areas of physiology and pharmacology for medical students, anaesthesia case problems would add some clinical context for undergraduates and could also provide a learning gradient based on the number of additional factors involved with the case. For example, ASA class II or III patient may provide a more complex case for medical students than an ASA class I patient. These are just a few of the ways in which anaesthesiology can contribute strongly to problem-based learning commonly used by Canadian medical schools.

\section{Exposure to Anaesthesiology Research to Increase Physician Scientists}

Another reason for the field of anaesthesiology to play a greater role in the undergraduate medical curriculum is to stimulate and feed research for the profession. Clinical innovation and scientific discovery are one of the hallmarks of any medical specialty and anaesthesiology should be no exception. ${ }^{5}$ Indeed, anaesthesiology has benefited tremendously from physician innovation from such pioneers as the late Dr Paul Janssen who was the founder of Janssen Pharmaceutica and the developer of over 80 pharmaceuticals. ${ }^{6}$

According to Bevan, anaesthesia has not been involved with the front runners of research such as molecular medicine, genomics and outcomes research. ${ }^{7}$ While this may be the historical nature of anaesthesia research, many questions remain as to the mechanism of action of inhaled anesthetics and genetic variation in response to anesthetic exposure. ${ }^{8}$ This has led to some exciting new research avenues that 
can impact the direction of the field. Such research may occur via basic science research, clinical safety outcomes research and also such emerging areas as nanotechnology in drug delivery. ${ }^{9}$ Indeed, the advancement of drug delivery through the maturation of nanotechnology may allow future anaesthesiologists to revisit many drugs which may have contained previous barriers to administration. It is such cutting edge application of nanotechnology that if conveyed to medical students may inspire and encourage them to choose anaesthesiology as a career. This is particularly true as today's generation of medical students tend to be drawn to specialties applying novel technologies. Anaesthesia could be one of these specialties and allow a new generation of anaesthesiologists the chance to break new ground in anesthetic care. Furthermore, stimulating medical student interest in anaesthesia research may help address the critical shortage of physician scientists in the field. ${ }^{10}$ This will be crucial in continuing to advance anaesthesiology as a leader in surgical safety and perioperative outcomes.

\section{Educating Students in Perioperative Medicine}

Anaesthesiologists are increasingly playing a larger role in comprehensive perioperative care. While many pre-clerkship medical students have the opinion that anaesthesiologists practice only in the operating room, their skills are frequently applied in pre-admission clinics, postoperative management and in critical care. Anaesthesiologists have long been experts in the simulation of technical procedures such as tracheal intubation and vascular access, and such simulation has led to improved intraoperative and perioperative patient safety. ${ }^{7}$ These skills are essential to many fields of medicine particularly in a surgical setting, but anaesthesiology educators are uniquely positioned to teach students about multifactorial components of perioperative care. In perioperative settings, much of what anaesthesiologists do is the treatment and prevention of organ dysfunction and as such are involved in some of the most comprehensive outcome studies. ${ }^{7}$ The tasks involved in perioperative care are ideal to train medical students in the skills that fall underneath the ever increasing range of anaesthesiologist activities. Moreover, in pre-clerkship, perioperative events may also provide a rich source of problem-based learning scenarios which could introduce concepts from surgery, physiology and pharmacology to name a few. Alternatively, preoperative preparation, risk evaluation and mitigation are skills that can most certainly be translated to other disciplines when planning the management of patients with a number of underlying diseases.

The modern medical curriculum has moved towards a problem-based learning model. Anaesthesiologists are ideally suited to educate in this manner and can provide clear clinical examples of pharmacology and physiology to enrich this learning process. In addition to augmenting the delivery of basic science curriculum, anaesthesiologists are well equipped to translate these concepts to clinical scenarios. Such involvement of anaesthesiologists in medical education may also expose students to potential research avenues and the direct impact that such research can have on patient care. Furthermore, anaesthesiologists are experts in perioperative care and can educate students on this topic in a way few other specialties can. By integrating more anaesthesiology educators into the undergraduate medical curriculum, not only will the quality of education improve but it may increase the number of students pursuing a career in the specialty.

\section{REFERENCES}

1. Sullivan P. Anaesthesia for medical students. Ottawa: DocuLink International; 1999.

2. Bradley JW. Can anaesthesiologists be effective teachersin the reformed curriculum? In:. Proceedings of the 12th World Congress of Anaesthesiologists. Montreal, 2000; 40-2.

3. Brull R, Bradley JW. The role of anaesthesiologists in Canadian undergraduate medical education. Can J Anesth 2001; 48:147-52.

4. Young H. Lack of pharmacological training causes overuse and misuse of drugs. CMAJ 2008; 178:276.

5. Orser BA, Miller DR. New opportunities for anaesthesia research in Canada. Can J Anesth 2002; 49:895-9.

6. Stanley TH, Egan, TD, Van Aken H. A Tribute to Dr Paul A.J. Janssen: Entrepreneur Extraordinaire, Innovative Scientist, and Significant Contributor to Anaesthesiology. Anaesthesia \& Analgesia 2008; 106:451-62.

7. Bevan DR. The future of academic anaesthesia departments in Canada. Can $\mathrm{J}$ Anesth 2006; 53:533-9.

8. Campagna JA, Miller KW, Forman SA. Mechanisms of actions of inhaled anesthetics. New Engl J Med 2003; 348:2110-24.

9. Hung O. Drug transformation: Advances in drug delivery systems. Can J Anesth 2006; 53:1074-7.

10. Schwinn DA, Balser JR. Anaesthesiology physician scientists in academic medicine: a wake-up call. Anaesthesiology 2006; 104:170-78. 